\title{
Oral hygiene habits and status of orthodontic patients attending the University of Pretoria, Oral and Dental Hospital
}

SADJ April 2021, Vol. 76 No. 3 p130 - p135

NL Buthelezi', TK Madiba ${ }^{2}$

\section{ABSTRACT}

\section{Background}

Most orthodontic patients struggle to maintain good oral hygiene during treatment.

\section{Aim}

To determine oral hygiene habits and status of patients undergoing fixed orthodontic treatment at University of Pretoria, Oral and Dental Hospital.

\section{Design}

A cross-sectional descriptive study.

\section{Material and methods}

A modified, validated, self-administered questionnaire was used, and clinical examinations were conducted using Orthodontic Plaque Index, Gingival index, and Bleeding index. The questionnaire sought to determine knowledge and practice patterns. Data analysis included frequencies and correlations using chi-square test, with a significance of $p<0.05$.

\section{Results}

Fifty patients participated with 34(68\%) being female with ages from 10 to 28 and a mean of 18,5 . Seventy percent avoided sticky foodstuff, $74 \%$ used mouthwash, 56\% flossed daily and $84 \%$ brushed twice daily. However, $82 \%$ consumed sugar containing drinks.

Clinical exam revealed an Orthodontic Plaque Index mean of 2.6, Gingival Index mean of 0.1 while the Bleeding

\footnotetext{
Author affiliations:

1. Noluthando L Buthelezi: , DipOH, AdvDipOH (Ortho), PGCHE, BEd Hons (TEPD), Department of Orthodontics, School of Dentistry, University of Pretoria, Pretoria, South Africa. ORCID Number: 0000-0002-3900-7707

2. Thomas K Madiba: B.Dent Ther, BDS, DHSM, MChD (Community Dentistry), Department of Community Dentistry, School of Dentistry, University of Pretoria, Pretoria, South Africa. ORCID Number: 0000-0002-0171-0595

Corresponding author: Thomas K Madiba

Department of Community Dentistry, School of Dentistry, University of

Pretoria, Pretoria, South Africa.

P.O. Box 21497, Valhalla, 0137

Email: thommy.madiba@gmail.com

Author contributions:

1. Noluthando L Buthelezi: Principal author - 50\%

2. Thomas K Madiba: Second author $-50 \%$
}

Index was 13.3 and $90 \%$ had normal gingiva. There was a significant difference in Gingival Index score between patients at age category 10-19 and 18-24 ( $p<0.05)$.

\section{Conclusions}

This study revealed a satisfactory oral hygiene status among patients at the institution with the majority of patients maintaining good oral hygiene practices. However, $82 \%$ consumed sugar sweetened beverages.

\section{Keywords}

Oral hygiene habits, orthodontic patients.

\section{BACKGROUND AND LITERATURE}

Orthodontic treatment is received by individuals with dentofacial anomalies and the treatment is done to improve their appearance. ${ }^{1}$ Treatment is undertaken using different techniques involving the use of fixed orthodontic appliances, removable appliances, and functional appliances.

The use of fixed orthodontic appliances (archwires and different ligating systems where orthodontic brackets are attached to the teeth) makes it difficult for the patients to keep their oral hygiene to an optimum level of cleanliness. ${ }^{2,3,4}$

Patients' oral hygiene is recognized as an important determinant for orthodontic treatment time and quality of the orthodontic treatment outcome. ${ }^{5}$ Poor oral hygiene attracts significant plaque accumulation around the brackets, ${ }^{6}$ and subsequent white spot lesions can occur rapidly, usually on the cervical and middle third of the buccal surfaces of bracketed teeth. ${ }^{7}$

Periodontal complications are observed in patients with poor oral hygiene during orthodontic treatment; the complications include gingivitis, gingival hyperplasia, gingival recession, periodontitis and more. ${ }^{8}$

As reported by one study, having good oral hygiene improves the success of the orthodontic treatment, as good oral hygiene promotes tooth movement and lowers the chances of oral diseases. ${ }^{9}$ 
Oral hygiene plays a very important role in orthodontics and can have a huge impact on the outcome or results of the treatment. ${ }^{10}$ Several studies have been conducted in assessing the status of oral hygiene amongst the patients who are undergoing fixed orthodontic treatment. A study conducted at the University of Benin Teaching Hospital in Nigeria where the oral hygiene status of fortythree orthodontic patients was assessed using the Simplified Oral hygiene Index, revealed that $62.8 \%$ of the patients exhibited good oral hygiene and $37.2 \%$ had fair oral hygiene while no patients were found with poor oral hygiene. ${ }^{11}$ This study did not include other indices such as the gingival index, orthodontic plaque index and bleeding index. The omission of these indices could lead to incorrect diagnosis, as it can happen that a patient can present with a low plaque index but show signs of gingivitis. The latter could indicate that the patient may have brushed efficiently prior to their orthodontic appointment, which could be interpreted as the patient having knowledge of what they need to do to maintain good oral hygiene but might not be practicing good oral hygiene religiously. Using this index only therefore cannot be seen as reliable and a definitive determinant of the patient's oral hygiene status.

The Oral Hygienist plays a very important role during orthodontic treatment in ensuring that the patient has all the information they need for different phases of the orthodontic treatment. ${ }^{5}$ Educating and motivating the patient who has orthodontic appliances to maintain good oral hygiene is necessary to decrease the risks of oral diseases. ${ }^{12}$ The risks can be decreased if both the practitioners (Orthodontist, Dentist and Oral Hygienist) and the patients are committed. It is also the responsibility of the patient to comply to the treatment objectives and the information and preventive measures given to him/her by the practitioner. $^{12}$

To the authors knowledge this study has never been undertaken for the institution and therefore it is important to assess the oral hygiene habits and status of orthodontic patients attending the University of Pretoria, Oral and Dental Hospital. Findings from the study will help the institution to put in measures necessary to ensure a satisfied experience of orthodontic patients.

\section{METHODOLOGY}

Ethical approval was obtained from the University of Pretoria, Faculty of Health Sciences Ethics committee (Ref 504/2015). No personal details of the patients were disclosed, and all information was strictly confidential and anonymous.

A cross-sectional descriptive study of patients undergoing fixed orthodontic treatment was conducted using a modified, validated, self-administered questionnaire ${ }^{13}$ and clinical examination of teeth and the gingiva. The clinical examinations of patients made use of Orthodontic Plaque Index ${ }^{14}(\mathrm{OPI})$, Gingival Index ${ }^{15}(\mathrm{Gl})$ and Bleeding Index ${ }^{16}(\mathrm{BI})$ which were recorded for each patient.

The validated questionnaire was adapted from a study by Atassi and Awartani (2010). It consisted of twentythree (23) questions that covered topics related to the knowledge and practice of patients concerning fixed or- thodontic treatment. The knowledge questions consisted of sixteen (16) items that sought to determine whether patients knew the reasons for having braces, whether they knew what types of foods to avoid and whether they had been to their dentist/oral hygienist for a professional cleaning. The questions were both close ended and open ended to determine the depth of patients' knowledge.

To determine the patient's practices, seven (7) questions were asked, and they sought to determine whether patients flossed their teeth, whether they brushed their teeth and the frequency per day and whether they used mouth rinse. These questions also consisted of both closed and open-ended questions.

\section{Clinical examination}

The use of clinical examination instruments (mouth mirror, periodontal probe, tweezers, and mouth retractor) was used to assess the oral hygiene status of the participants.

The process of clinical examination was employed using the following indices:

\section{Bleeding index}

Each tooth present was gently probed with a periodontal probe at six sites (mesial, mid, and distal on both buccal and lingual surfaces). ${ }^{16}$ Bleeding was scored as present or absent and the number of sites where bleeding was present were recorded.

The number of sites where bleeding was recorded were divided by the total number of available sites in the mouth and multiplied by 100 to express the bleeding index as a percentage.

\section{Gingival index}

The criteria used for gingival assessment was done according to the work of Löe and Silness (1963) where the marginal and interproximal tissues were assessed and scored using the scores below. ${ }^{15}$

$0=$ Normal gingiva .

$1=$ Mild inflammation - slight change in colour and slight edema but no bleeding on probing.

2 = Moderate inflammation - redness, edema and glazing, bleeding on probing.

$3=$ Severe inflammation - marked redness and edema, ulceration with tendency to spontaneous bleeding.

\section{Orthodontic plaque index}

The Orthodontic Plaque Index (OPI) is a special index for patients with fixed orthodontic appliances. The OPI focuses on the tooth area in the immediate vicinity of the bracket, since additional and relatively inaccessible plaque niches arise at these sites. ${ }^{14}$

To record the OPI, the dentition was divided into sextants. A disclosing agent (2-Tone) was used to disclose plaque. 
The plaque accumulation on each tooth surface adjacent to the bracket base was evaluated (mesial, distal, occlusal/incisal, and cervical) and recorded in the clinical data collection sheet.

0 : No plaque deposits on the tooth surfaces surrounding the bracket base.

1: Plaque deposits on one tooth surface at the bracket base.

2: Plaque deposits on two tooth surfaces at the bracket base.

3: Plaque deposits on three tooth surfaces at the bracket base.

4: Plaque deposits on four tooth surfaces at the bracket base and/or gingival.

One examiner conducted the clinical examinations for all three indices. Ten subjects who volunteered to participate were examined on two occasions using the three indices $(\mathrm{BI}, \mathrm{Gl}$, and $\mathrm{OPI})$ to establish intra-examiner reliability.

The Kappa test was used to analyze the intra-examiner reliability and scored 71.4 percent, 79.7 percent, and 81 percent for GI, PI, and OPI respectively. All patients were seen during their regular recall appointments. The clinical assessment and the filling in of the questionnaire form took approximately twenty minutes (20 minutes) per participant.

The study was performed in the Postgraduate Clinic of the Department of Orthodontics at the Oral and Dental Hospital (University of Pretoria) and was conducted over the period of 21 weeks in 2018. The department had two full time registrars that were treating 160 patients among themselves that were undergoing fixed orthodontic treatment. With the population of 160 and a confidence interval of $95 \%$, a minimum sample size of 47 was deemed to be sufficient. The selection criteria were:

- Treatment that consisted of full mouth fixed orthodontic appliances that had been in place for at least six months.

- No systemic diseases.

- No history of taking antibiotics for the last three months.

- No treatment by an Oral hygienist any time during the month preceding the study.

Fifty (50) patients that fitted the criteria and were able to come when the registrars had sessions which was Mondays, Tuesdays and Wednesday and agreed to take part in the study. Quota sampling was used to choose the participants. This was done through identifying fifty (50) patients who were meeting the selection criteria as stated above. The patients who met the selection criteria were then conveniently sampled as they came in.

Data was analyzed with SPSS Version 25. Descriptive and analytical statistical tests were done, and the level of confidence was set at $95 \%$. Chi square test was used to evaluate the association between variables: Level of significance was be set at $p<0.05$.

\section{RESULTS}

The study had 50 participants with 34 (68\%) of them being female and 16 (32\%) of them being male. The age ranged from 10 to 28 with a mean age of 18,5.

The knowledge and practice patterns of the study participants are shown in Table 1 below.

\begin{tabular}{|c|c|c|}
\hline Knowledge & Yes $(n / \%)$ & No $(n \%)$ \\
\hline Do you know why you have braces? & $49(98)$ & $1(2)$ \\
\hline $\begin{array}{l}\text { Where you shown how to clean your teeth after } \\
\text { your braces were done? }\end{array}$ & $45(90)$ & $5(10)$ \\
\hline Do you eat hard sticky food? & $15(30)$ & $35(70)$ \\
\hline $\begin{array}{l}\text { Do you know why you should avoid hard } \\
\text { sticky foods? }\end{array}$ & $48(96)$ & $2(4)$ \\
\hline Are you happy with your gums? & $35(70)$ & $15(30)$ \\
\hline \multicolumn{3}{|l|}{ Practices } \\
\hline Do you use interdental brushes under your braces & $25(50)$ & $25(50)$ \\
\hline Do you use a mouthwash? & $37(74)$ & $12(24)$ \\
\hline Have you had your teeth cleaned by a professional & $40(80)$ & $10(20)$ \\
\hline Do you drink sugar containing drinks & $41(82)$ & 7 (14) \\
\hline \multicolumn{3}{|l|}{ How often do you brush your teeth (n/\%) } \\
\hline \multicolumn{3}{|l|}{ Once a day $3(6)$} \\
\hline \multicolumn{3}{|l|}{ Twice a day $42(84)$} \\
\hline \multicolumn{3}{|l|}{ Three times a day 5 (10) } \\
\hline \multicolumn{3}{|l|}{ How many times do you use a mouth wash ( $\mathrm{n} / \%)$} \\
\hline \multicolumn{3}{|l|}{ Once a day $22(44)$} \\
\hline \multicolumn{3}{|l|}{ Twice a day $14(28)$} \\
\hline \multicolumn{3}{|l|}{ Three times a day 2 (4) } \\
\hline \multicolumn{3}{|l|}{ How often do you floss ( $\mathrm{n} / \%$ ) } \\
\hline \multicolumn{3}{|l|}{ I don't floss 8 (16) } \\
\hline \multicolumn{3}{|l|}{ Once a day $28(56)$} \\
\hline \multicolumn{3}{|l|}{ Twice a week $13(26)$} \\
\hline Four times a week 1 (2) & & \\
\hline
\end{tabular}

Table 1 above indicates that most of the patients had knowledge about orthodontic treatment and were aware what food to eat and had the knowledge that sticky and sugary drinks were not good for them. However as far as the practice was concerned $82 \%$ of the patients were consuming sugar containing drinks. More than half (56\%) of the patients were flossing once a day, $84 \%$ were brushing twice a day and $80 \%$ of the patients had their teeth cleaned by the professional oral health practitioner.

\begin{tabular}{l|c|c|c|}
$\begin{array}{l}\text { Table 2. Mean Gingival, Bleeding and Orthodontic indices scores of } \\
\text { participants } \mathrm{n=50.}\end{array}$ & $\begin{array}{c}\text { Gingival } \\
\text { index }\end{array}$ & $\begin{array}{c}\text { Bleeding } \\
\text { index }\end{array}$ & $\begin{array}{c}\text { Orthodontic } \\
\text { index }\end{array}$ \\
\hline Mean & 0.1 & 13.3 & 2.6 \\
\hline Std deviation & 0.4 & 15.9 & 0.7 \\
\hline Minimum & 0 & 0 & 1 \\
\hline Maximum & 3 & 83 & 4 \\
\hline
\end{tabular}

The mean gingival index is 0.1 and it shows that most patients had normal gingiva with no inflammation. The bleeding index mean was 13.3 which indicated that most patients had bleeding with 1 patient with a very high score of 83. As far as the OPI was concerned the mean was found to be 2.6 which means that there was plaque found on two to three surfaces of the bracket bases.

When evaluating the association between two indices scores $(\mathrm{Bl}$ and $\mathrm{OPI})$ and age and gender there were no 
significant differences ( $p>0.05)$. There were also no significant differences between gender and age as far as frequency of brushing, flossing, use of mouth rinse, appearance of gums and consuming sweetened drinks (p>0.05).

There was also no correlation between age and $\mathrm{Gl}$ score $(p=0.21)$. However, there was a significant correlation between age and $G$ l score $(p=0.02)$ with more than half of patients in the age category 10-19 years of age having a zero score (58\%) as compared to $16 \%$ of 24-28. See Table 3. It was also interesting to realise that only $8 \%$ had a score of 1 and $92 \%$ of the patients had a score of zero which meant that more than $90 \%$ of the patients had normal gingiva. See Table 3 below.

\section{DISCUSSION}

The study had 50 participants with 34 (68\%) of them being female and 16 (32\%) of them being male. This could be explained by the fact that most females are conscious of their overall appearance and the possibility that the female patients were easily and conveniently available during the period of data collection. Orthodontic treatment aims at achieving aesthetic harmony, functional efficiency, and structural balance of the dentofacial region. ${ }^{17}$

Females patients could be well motivated to have this procedure done on them as it could lead to improved personal appearance. The age ranged from 10-28 years with a mean age of 18.5. The latter finding could be due to many reasons why patients in younger years seek orthodontic treatment, this inter alia includes the desire to have better dental appearance, straight teeth, selfconfidence and social acceptance. . $^{18,19,20}$

In testing the practices and the knowledge of the patients, the findings indicated that most patients were knowledgeable and well informed about diet during orthodontic treatment (see Table 1). This might be because oral health care workers in the department of orthodontics in the institution availed themselves to educate and motivate patients before and after the installation of fixed orthodontic brackets, a fact that was reported by patients.

While patients knew what food to consume and what to avoid, $82 \%$ of patients were consuming sugary containing drinks which was higher than a similar study. ${ }^{13}$ This percentage is very high and indicates that while patients reported that they knew what foodstuffs to take and the fact that sugar containing drinks was not good for them, their practices did not reflect their knowledge. This was not surprising as literature indicate that knowledge does not always translate into changes in behaviour or attitude, but that changes in behaviour are influenced by conditions in which people live. ${ }^{21}$
Many health promotional activities fail because health professionals assume that by providing health education, knowledge will increase, and this would result in change of behaviour and attitudes. ${ }^{21}$

The high consumption of sugar sweetened beverages by most patients is a cause of concern and therefore the oral health workers treating these patients need to reinforce that aspect of knowledge.

According to the findings $56 \%$ of the patients indicated that they flossed once a day. Again, the oral health care workers in this department should enforce the importance of flossing once a day to the patients. A high percentage of patients (84\%) indicated that they brushed twice a day which is what is recommended during patient education. Only $6 \%$ of the patients brushed once a day and these patients must be reminded of the significance of brushing at least twice a day.

It is equally important that patients who are on fixed orthodontic treatment should visit an oral hygienist or a dentist to have their teeth professionally cleaned and checked up to manage and eliminated the occurrence of oral diseases. The findings indicate that $80 \%$ of the patients have visited the oral hygienist or dentist to have their teeth scaled and polished. This means $20 \%$ of the subjects have not had their teeth professionally cleaned. These patients need to be booked for professional cleaning and reminded of the importance of having their teeth professionally cleaned regularly.

The study found that a high percentage (90\%) of patients undergoing fixed orthodontic treatment in the department of orthodontics at the Oral and Dental Hospital had normal gingiva. This finding contrasts with a study conducted among orthodontic patients in King Saudi Hospital. ${ }^{13}$ In this study $40 \%$ of the patients indicated to have a fair oral hygiene while $60 \%$ were indicated to have poor oral hygiene.

The higher percentage in this study could be explained by the fact that the study sample consisted of a higher percentage of female patients and females in general are known to have better hygiene standards compared to males. In the King Saudi Hospital study there was had a higher percentage of male participants (64\%) and a low number of female participants (36\%).

The high percentage in this study is also an indication that patients took the oral hygiene instruction that was given to them seriously and practised them. This is evidenced by the fact that $90 \%$ of the patients admitted that they were shown how to clean their teeth after the brackets were bonded and the fact that the majority of the patients brushed their teeth twice a day.

\begin{tabular}{|c|c|c|c|c|c|c|}
\hline \multirow[t]{2}{*}{ Age category (years) } & \multicolumn{6}{|c|}{ Gingival Index score (n/\%) } \\
\hline & 0 & 1 & 2 & 3 & Total $(n / \%)$ & $P$ value \\
\hline $10-19$ & $29(58)$ & $2(4)$ & $0(0)$ & $0(0)$ & $31(62)$ & \multirow[t]{4}{*}{0.02} \\
\hline $20-23$ & $9(18)$ & $2(4)$ & $0(0)$ & $0(0)$ & $11(22)$ & \\
\hline $24-28$ & $8(16)$ & $0(0)$ & $0(0)$ & $0(0)$ & $8(16)$ & \\
\hline Total $(n / \%)$ & $46(92)$ & $4(8)$ & $0(0)$ & $0(0)$ & $50(100)$ & \\
\hline
\end{tabular}


Even though the OPI revealed a mean of 2.6 which indicate that for most patients there were plus minus three surfaces in the vicinity of a bonded bracket that were covered by dental plaque. This can hypothetically give the impression that most patients have poor oral hygiene, hence this study combined OPI with other indices to get better understanding of the oral hygiene status of the patient.

A study by Ajayi E and Azodo C (2014) only used simplified plaque index and did not use other indices such as the gingival index, orthodontic plaque index and bleeding index. ${ }^{11}$ This was somewhat skewed as a patient can have a $10 \%$ or less plaque index but present with signs of gingivitis which indicates that the patient may have brushed efficiently prior to their orthodontic appointment.

Using this index only therefore cannot really predict the efficacy of the patient's oral hygiene practices. The only conclusion that can be drawn from only using simplified plaque index is whether patient have knowledge on good oral hygiene regimen. The fact that patients in this study had about three surfaces covered with plaque might be an indication of the difficulty in cleaning around the brackets which is a common finding with patients undergoing Orthodontic treatment. ${ }^{13,22,23}$ The encouraging fact is that most patients had normal gingiva which means that reinforcement of oral hygiene practices can solve the problem.

The study performed by Atassi F \& Awartani F (2010) depicted that motivating patients to maintain good oral hygiene practices can improve patient homecare regimen. ${ }^{13}$ The presence of an oral hygienist who is patientcentred in an orthodontic practice can therefore help to improve the oral hygiene status and knowledge of patients with fixed orthodontic treatment. ${ }^{24}$

There was a significant association between age and Gl score $(p=0.02)$ with more than half of patients in the age category 10-19 years of age having a zero score $(58 \%)$ as compared to $16 \%$ of $24-28$. The finding in this study were similar to patients that were treated at university of Benin in Nigeria that found that the subjects aged between 11-20 years old exhibited a significantly higher level of good and fair oral hygiene status. ${ }^{11}$ One would have thought that the older ages would do better in terms of gingival health as their practices are expected to be better because it is assumed their understanding is higher.

\section{CONCLUSIONS AND RECOMMENDATIONS}

This study revealed a satisfactory oral hygiene status among this sample with $90 \%$ of the patients having normal gingiva, more than half flossing daily, used mouth wash and $84 \%$ brushing twice a day. Although this was the case patients had three surfaces on average having plaque around orthodontic brackets, a common occurrence in patients with orthodontic brackets.

There were no significant differences between gender and age as far as frequency of brushing, flossing, use of mouthwash, appearance of gums and consuming sweetened drinks. There was also no association between age and
Gl score However this study showed a significant association between age and Gl score, with more than half of patients in the age category 10-19 years of age having a zero score (58\%) as compared to $16 \%$ of $24-28$ ages. Although the majority of patients in this study reported that they were told what food to eat and how to practice oral hygiene $82 \%$ were consuming sugar sweetened beverages.

The study revealed that knowledge does not translate into change of behaviour and therefore the oral health care workers should continue educating and motivating these patients to maintain their oral health and providing recommendations for oral home care aids to enhance their compliance. ${ }^{25}$

They should encourage the patients to gain an understanding of what their responsibilities are and to understand they are partners in their orthodontic treatment and have an opportunity to improve and then maintain good oral healthcare themselves. The staff must also understand that oral hygiene programs won't be effective unless the team accept the responsibility for motivating their patients.

\section{Limitations}

This study is limited by the cross-sectional study design, and causality cannot be inferred. Response acquiescence is common in questionnaires that tend to determine habits that are considered taboo or have negative connotations. Despite the limitations, the current study provided useful information that may inform future oral health education approaches of patients at the institution.

\section{Financial support and sponsorship}

None.

\section{Declaration}

No conflict of interest declared.

\section{References}

1. Shaw WC, O'Brien KD, Richmond S. Quality control in orthodontics: factors influencing the receipt of orthodontic treatment. Br Dent J. 1991; 170(2): 66-8.

2. Derks A, Kuijpeers-Jagtman AM, Frencken JE, Martin A, Hof V, Katsarose C. Caries preventative measures used in orthodontic practices: An evidence-based decision. AJO. 2007; 132(2): $165-70$.

3. Zachrisson BU, Alnaes L. Periodontal condition in orthodontically treated and untreated individuals. I. Loss of attachment, gingival pocket depth and clinical crown height. Angle Orthod. 1973; 43(4): 402-11.

4. Alexander SA. Effect of orthodontic attachments on the gingival health of permanent second molars. Am $\mathrm{J}$ Orthod Dentofacial Orthop. 1991; 100(4): 337-40.

5. Duncan Y. Brown , Terry D. Carlyle, Darren Isfeld. Systematic evaluation of patient oral hygiene for orthodontic patients. AJO. 2009; 1(1): 1-8.

6. Øgaard B, Rølla G, Arends J. Orthodontic appliances and enamel demineralization Part 1. Lesion development. Am J Orthod Dentofacial Orthop. 1988; 94(1): 68-73. 
7. Chang HS, Walsh LJ, Freer TJ. Enamel demineralization during orthodontic treatment. Aetiology and prevention. Aust Dent J. 1997; 42(5): 322-7.

8. Morrow D, Wood DP, Speechley M. Clinical effect of subgingival chlorhexidine irrigation on gingivitis in adolescent orthodontic patients. Am J Orthod Dentofac Orthop. 1992; 101 (5): 408-13.

9. Kharsa MAl. The importance of oral hygiene in orthodontic treatment. The Orthodontic Cyber Journal. 2008; 1(1): 1-9.

10. Skidmore KJ, Brook KJ, Thomson WM, Harding WJ. Factors influencing treatment time in orthodontic patients. AJO. 2006; 129(2): 230-8

11. Ajayi EO. Oral hygiene status among orthodontic patients attending, journal of dental health, oral disorders and therapy. 2014; 1(4): 1-4.

12. Bardal PAP, Olympio KPK, Bastos JRM, Henriques JFC, Buzalaf MAR. Education and motivation in oral health-preventing disease and promoting health in patients undergoing orthodontic treatment. Dental Press J Orthod. 2011; 16(3): 95-102.

13. Atassi F, Awartani F. Oral hygiene status among orthodontic patients. J Contemp Dent Pract. 2010; 11(4): 25-32.

14. Heintze SD, Jost-Brinkmann PG, Finke C, Miethke RR. Orthoplaque Index. In: Oral health for the orthodontic patient. Chicago: Quintessence. 1999; 67-70.

15. Löe $H$. The gingival index, the plaque index and the retention index systems. The Journal of Periodontology. 1967; 38(6): 610-6.

16. Newbrun E. Indices to measure gingival bleeding. Journal of periodontology. 1996; 67(6): 555-61.

17. Karimi-Afshar M, Torabi M, Safarian F, Dehghan MA, KarimiAfshar M. Effect of orthodontic treatments on quality of life in adolescents. Journal of Oral Health and Oral Epidemiology. 2018; 7(4): 198-204.
18. Abdullah AA, Yassin ZM, Zamzam N. Reasons for seeking orthodontic treatment. A Pilot Study. Annals of Dentistry University of Malaya. 2001; 8(1): 13-9.

19. Al Fawzan A. Reasons for seeking orthodontic treatment in Qassim region: A Pilot Study. International Dental Journal of Students Research. 2013; 1: 58-62.

20. Jay P, Goje SK, Narayan K, Riddhi P, Chinmay D, Aakash S. Impact of orthodontic treatment needs on Oral health-related quality of life among the early adults: a questionnaire study. Journal of International Oral Health. 2018; 8(12): 1095-100.

21. Kay E, Vascott D, Hocking A, Nield H, Dorr C, Barrett $H$. A review ofapproaches for dental practice teams for promoting oral health. Community Dent Oral Epidemiol. 2016; 44: 313-30.

22. Geiger AM, Wasserman BH, Turgeon LR. Relationship of occlusion and periodontal disease. 8. Relationship of crowding and spacing to periodontal destruction and gingival inflammation. J Periodontol. 1974; 45(1): 43-9.

23. Lundström F, Hamp SE, Nyman S. Systematic plaque control in children undergoing long-term orthodontic treatment. Eur $\mathrm{J}$ Orthod. 1979; 2(1): 27-39.

24. Impellizzeri A, Samà R, Roberto Di Giorgio MD, Galluccio G. Control of oral hygiene in the orthodontic patient: comparison between public facility and private practice. Annali di Stomatologia. 2018; 9(1): 43-52.

25. Firth F, Bennani F, Stacknik S, Farella M. Orthodontic patient co-operation: A review of the clinician's role in predicting and improving patient compliance. Australasian Orthodontic Journal. (2019; 35: 1-5

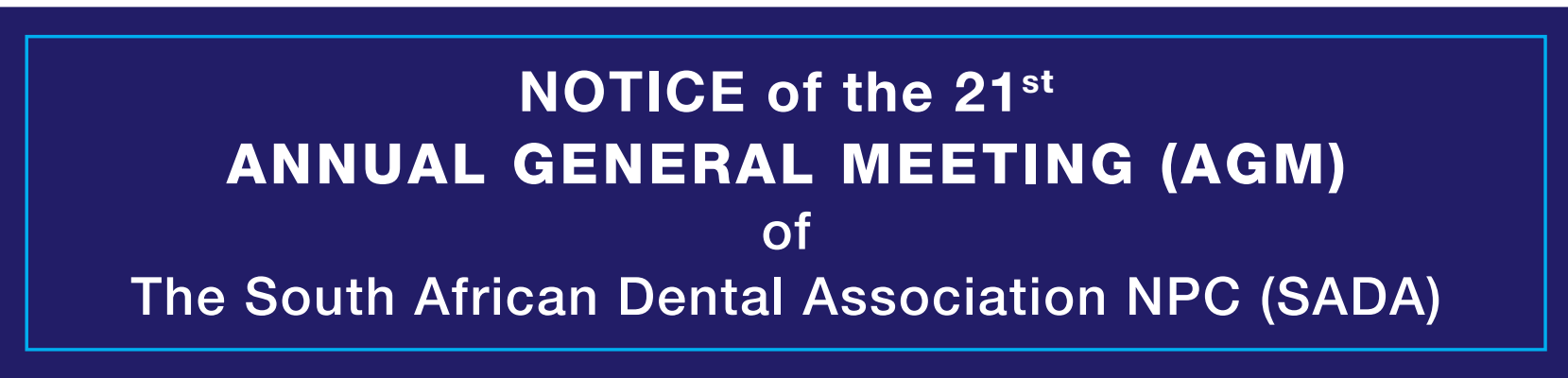

Notice is hereby given that the $21^{\text {st }}$ Annual General Meeting of Members (AGM) of The South African Dental Association (SADA) NPC, will be held on Thursday, 10 June 2021 at 18h00, which will be conducted virtually and electronically on this date through the Zoom virtual meeting platform or similar digital platform. The Agenda with any supporting documents for the meeting will be posted on the SADA website.

SADA is your Association and your voice counts.

\section{KC Makhubele}

Chief Executive Officer

April 2021

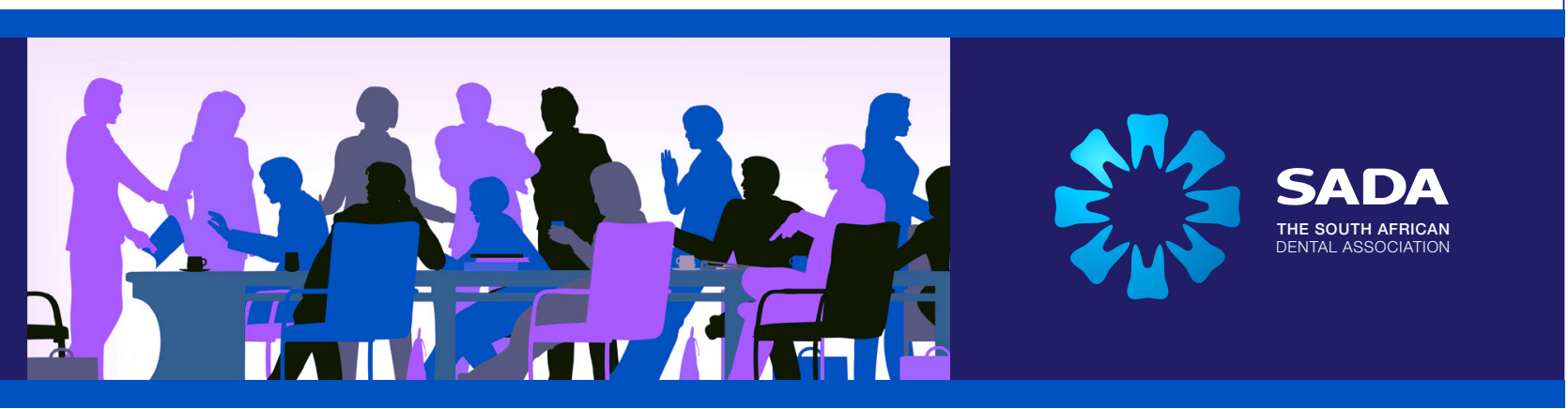

